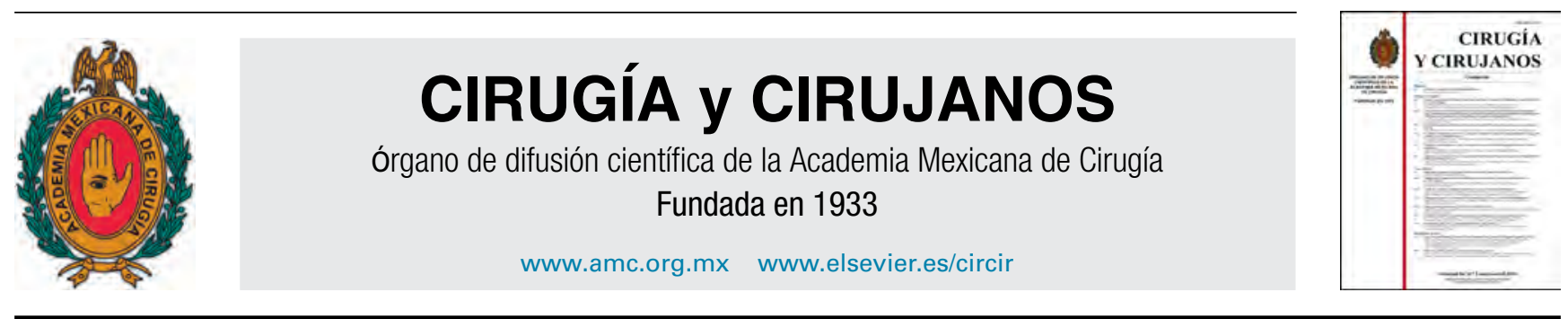

CASO CLÍNICO

\title{
Malrotación intestinal en adulto asociada a vólvulo intestinal
}

\section{Ernesto Hernando-Almudía,*, Rafael Cerdán-Pascual ${ }^{a}$, Cristina Vallejo-Bernad ${ }^{a}$, Joaquín Martín-Cuartero ${ }^{\mathrm{b}}$, María Sánchez-Rubio ${ }^{\mathrm{a}}$ y Carmen Casamayor-Franco ${ }^{\mathrm{a}}$}

\author{
a Servicio de Cirugía General, Hospital Universitario Miguel Servet, Zaragoza, España \\ b Servicio de Radiología, Hospital Universitario Miguel Servet, Zaragoza, España
}

Recibido el 4 de abril de 2016; aceptado el 17 de mayo de 2016

Disponible en Internet el 23 de junio de 2016

\section{PALABRAS CLAVE \\ Malrotación \\ intestinal; \\ Vólvulo intestinal; \\ Obstrucción intestinal}

\section{KEYWORDS}

Intestinal

malrotation;

Intestinal volvulus;

Intestinal obstruction

\begin{abstract}
Resumen
Antecedentes: La malrotación intestinal es una anomalía congénita de la rotación y fijación intestinal, que se presenta generalmente en la edad neonatal.

Objetivo: Descripción de un caso clínico asociado a un cuadro oclusivo agudo.

Caso clínico: Presentamos el caso de una mujer de 46 años, previamente asintomática, atendida por un cuadro de obstrucción intestinal, con el hallazgo radiológico y quirúrgico de una ausencia de rotación intestinal.

Conclusiones: La malrotación intestinal en el adulto, frecuentemente es asintomática, y se diagnostica de una forma casual, en el curso de una exploración radiológica realizada por otra causa. De forma infrecuente puede diagnosticarse en adultos, asociado a un cuadro de abdomen agudo.

(C) 2016 Academia Mexicana de Cirugía A.C. Publicado por Masson Doyma México S.A. Este es un artículo Open Access bajo la licencia CC BY-NC-ND (http://creativecommons.org/licenses/bync-nd/4.0/).
\end{abstract}

Adult intestinal malrotation associated with intestinal volvulus

\begin{abstract}
Background: Intestinal malrotation is a congenital anomaly of the intestinal rotation and fixation, and usually occurs in the neonatal age.

Objective: Description of a clinical case associated with acute occlusive symptoms.

Clinical case: A case of intestinal malrotation is presented in a previously asymptomatic woman of 46 years old with an intestinal obstruction, with radiology and surgical findings showing an absence of intestinal rotation.
\end{abstract}

\footnotetext{
* Autor para correspondencia: Avenida de Madrid 191, 4B, 50017 Zaragoza, España; Tel.: +34 $976532171 /+34617923385$.

Correo electrónico: ernestohernando@gmail.com (E. Hernando-Almudí).
} 
Conclusions: Intestinal malrotation in adults is often asymptomatic, and is diagnosed as a casual finding during a radiological examination performed for other reasons. Infrequently, it can be diagnosed in adults, associated with an acute abdomen.

(c) 2016 Academia Mexicana de Cirugía A.C. Published by Masson Doyma México S.A. This is an open access article under the CC BY-NC-ND license (http://creativecommons.org/licenses/bync-nd/4.0/).

\section{Antecedentes}

El término malrotación intestinal puede definirse como: un defecto en la rotación normal y fijación del intestino durante el desarrollo fetal. Abarca una gran variedad de anomalías que pueden permanecer asintomáticas toda la vida o acompañarse de un abdomen agudo, generalmente en forma de vólvulo intestinal asociado ${ }^{1}$.

La alteración del desarrollo embriológico intestinal puede ocurrir en cualquiera de sus fases, y podemos agruparlas según el estadio de desarrollo en el que ocurran en ${ }^{1}$ :

1. No rotación: en ella el intestino delgado se sitúa a la derecha del abdomen. El íleon distal cruza la línea media para alcanzar el ciego, el cual se sitúa en la línea media.

2. Rotación incompleta: el intestino ocupa una posición intermedia entre la no rotación y la disposición normal.

3. Rotación inversa: el duodeno cruza por delante de la arteria mesentérica superior, y el colon va por detrás de esta. Así, el duodeno está delante, después la arteria mesentérica superior y, por detrás de esta, el colon transverso.

En general la malposición intestinal por sí misma no produce problemas, pero habitualmente se asocia a una mala fijación intestinal, lo que puede predisponer al vólvulo intestinal.

\section{Caso clínico}

Paciente de 46 años de edad, sin antecedentes de interés, que acude a urgencias por cuadro de vómitos de repetición y ausencia de deposición de 3 días de evolución. Previamente había acudido en 2 ocasiones al centro hospitalario, sin causa orgánica a su cuadro. Se acompaña en esta ocasión de dolor abdominal de inicio epigástrico, y posteriormente se hace difuso. A la exploración, el abdomen esta distendido, ligeramente doloroso y sin irritación peritoneal.

El hemograma es normal, la bioquímica presenta un ligero aumento de urea y creatinina, y una PCR de 2,4. En la radiología simple de abdomen existe una dilatación de asas del intestino delgado, compatible con un cuadro suboclusivo.

La paciente ingresa en el servicio de digestivo, y ante la exacerbación del dolor en las horas siguientes, se solicita una tomografía axial computada (TAC) abdominal, en la que se aprecia dilatación de asas de intestino delgado en el hemiabdomen derecho, desplazando el ciego a una posición posterior y central (fig. 1). Existe, además, abundante líquido libre indicativo de sufrimiento de asas y agrupación

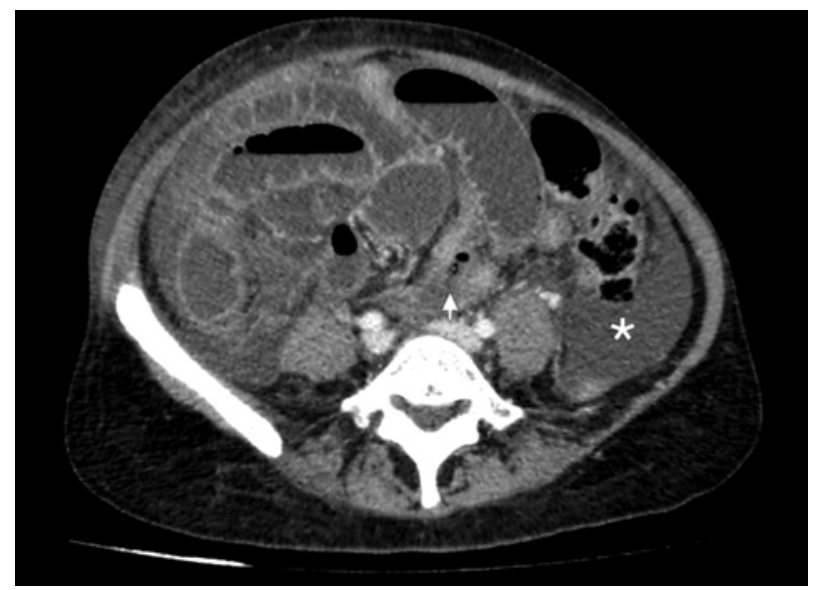

Figura 1 Imagen axial de la tomografía computada abdominal con contraste intravenoso. Dilatación de asas de delgado y presencia de líquido ascítico (asterisco). Posición anormal del ciego, localizado en línea media (flecha).

focal de vasos mesentéricos, sugestivos de hernia interna o torsión (fig. 2), por lo que el radiólogo sugirió como primera opción la de hernia interna pericecal. Retrospectivamente se observaba una inversión de la posición de los vasos mesentéricos (fig. 3).

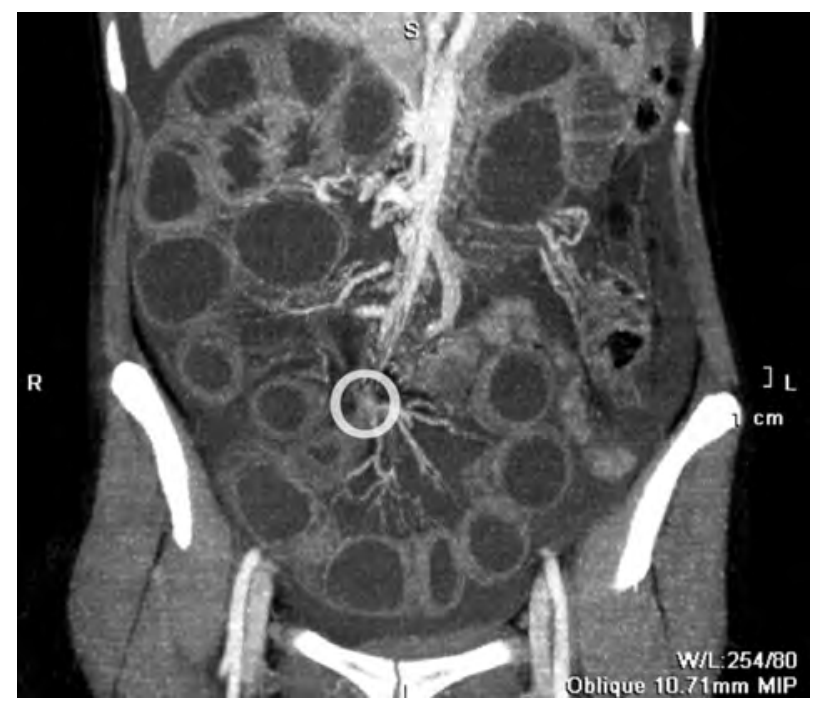

Figura 2 Reconstrucción coronal de la tomografía computada abdominal con contraste intravenoso. Dilatación generalizada de asas de delgado y presencia de líquido ascítico. Vasos mesentéricos agrupados a nivel del eje del vólvulo (círculo), responsable del cuadro oclusivo. 


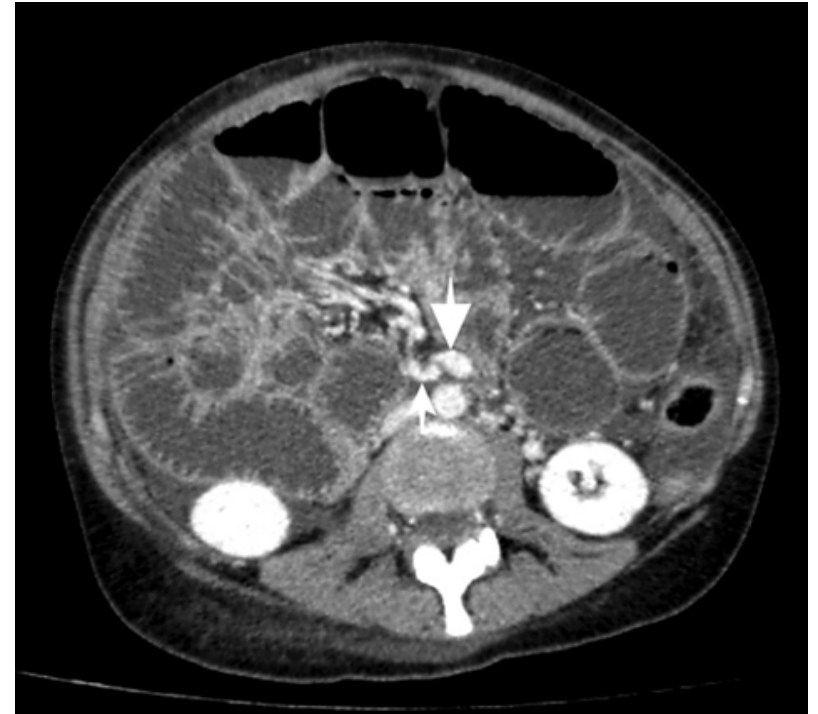

Figura 3 Imagen axial de la tomografía computada abdominal con contraste intravenoso. Inversión de la posición de los vasos mesentéricos, con artería mesentérica superior (flecha fina) localizada a la derecha de la vena mesentérica superior (flecha gruesa).

Se solicita al servicio de cirugía para su valoración, y a la exploración, la paciente presenta un abdomen muy distendido, doloroso, de forma difusa y escaso peristaltismo, con importante afectación del estado general.

Se le interviene quirúrgicamente de forma urgente, realizándose laparotomía exploradora, hallándose de inicio una isquemia intestinal irreversible de $150 \mathrm{~cm}$ de íleon, y un gran saco herniario en hemiabdomen derecho, con el ciego en posición medial y casi un litro de líquido intraabdominal.

Se abre y reseca el saco herniario que contiene yeyuno dilatado, pero no isquémico, y se comprueba la existencia de una malrotación intestinal con salida del ángulo de Treitz a la izquierda de los vasos mesentéricos y un vólvulo intestinal (figs. 4 y 5).

Se realizó exéresis de la mayor parte del saco mesentérico y la resección de los $150 \mathrm{~cm}$ de intestino afecto,

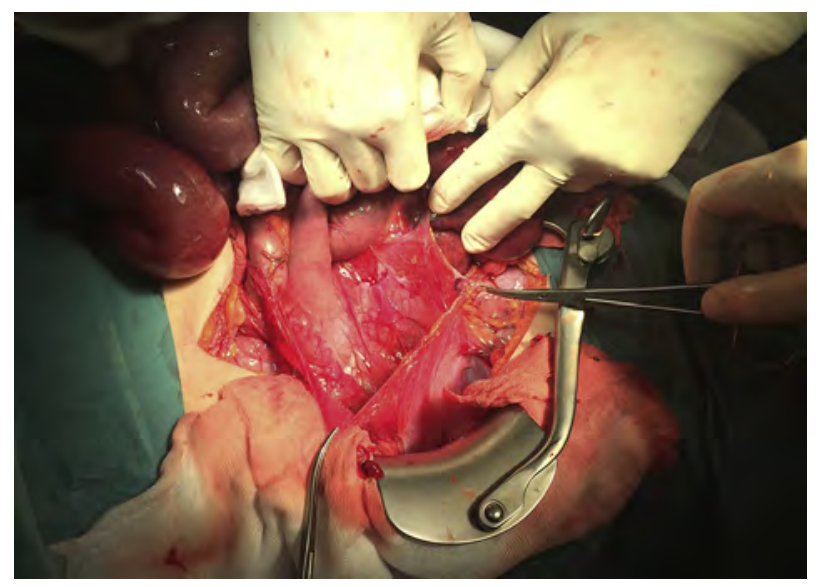

Figura 4 Saco mesentérico abierto y salida del yeyuno a nivel del ángulo de Treitz.

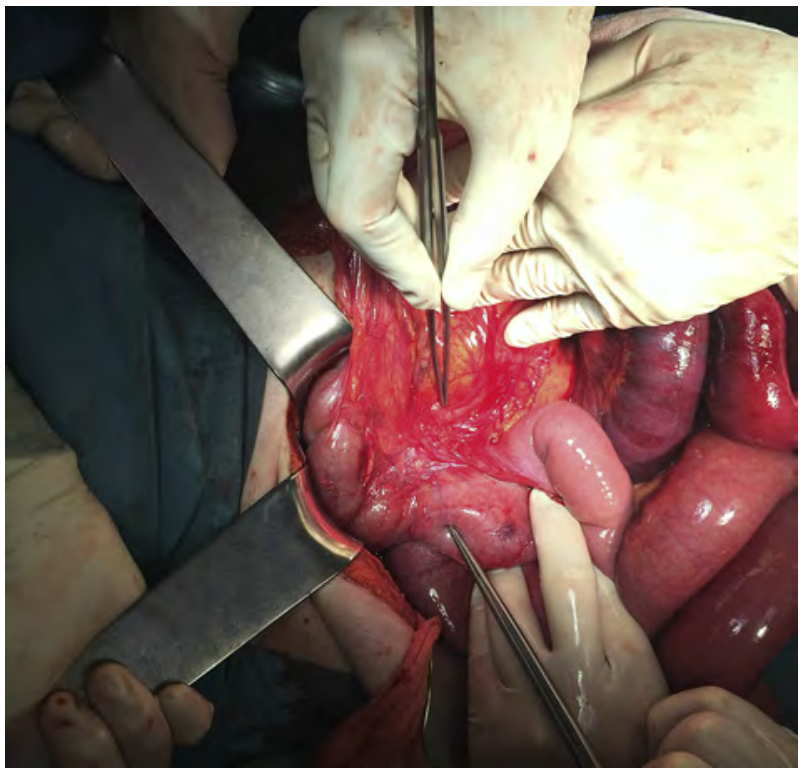

Figura 5 Posición anómala de los vasos mesentéricos.

quedando $300 \mathrm{~cm}$ por encima y $16 \mathrm{~cm}$ de íleon terminal, con anastomosis latero-lateral isoperistáltica manual.

El postoperatorio transcurrió con normalidad, siendo dada de alta a los 7 días de la cirugía.

\section{Discusión}

La malrotación intestinal está determinada por una interrupción o alteración del desarrollo embriológico del intestino medio, en cualquiera de sus fases, el cual se divide en 2 partes por el eje de la arteria mesentérica superior. La parte proximal, futura asa duodeno-yeyunal, se localiza por encima y anterior a la arteria mesentérica superior. La distal es posterior e inferior a la arteria, y dará lugar a íleon, colon ascendente y transverso proximal.

La incidencia en adultos se estima en un $0,2 \%^{2}$, y es habitualmente asintomática, diagnosticándose de forma casual en el curso de una exploración radiológica realizada por otra causa. El diagnóstico en adultos es difícil, dada su baja incidencia y la TAC es la técnica de elección ${ }^{3,4}$.

Lo característico de la TAC es la posición medial del ciego y lateral de las asas de delgado, e inversión de los vasos mesentéricos. En nuestro caso, complicado con un vólvulo, dilatación de asas de delgado, líquido libre y agrupación de vasos mesentéricos, similar a un arremolinamiento de vasos.

En otras ocasiones refieren síntomas crónicos con episodios de dolor abdominal y vómitos, incluso evidencia de malnutrición asociada a una malabsorción, debida a un vólvulo crónico intermitente. La incidencia de vólvulo intestinal es elevada (33\% de los casos), independientemente de la edad, lo que supone un abdomen agudo que debe tratarse de forma urgente ${ }^{5}$.

Definir el tipo exacto de malrotación tiene poco interés clínico ya que todas predisponen al vólvulo, lo importante es diagnosticar la existencia de esta. El tratamiento es quirúrgico, procediendo a desvolvular las asas de intestino, 
liberar las membranas que fijan a estas, separar el meso común y una apendicectomía, dado que la situación anormal del ciego dificulta un diagnóstico posterior de apendicitis.

\section{Conclusiones}

La malrotación intestinal en el adulto es un cuadro clínico, en la mayoría de las ocasiones asintomático. Su diagnóstico suele ser casual en el curso de una exploración radiológica, habitualmente una tomografía computada realizada por otro motivo. De forma infrecuente se asocia a un cuadro de abdomen agudo, siendo el diagnóstico en el curso de una laparotomía.

\section{Responsabilidades éticas}

Protección de personas y animales. Los autores declaran que para esta investigación no se han realizado experimentos en seres humanos ni en animales.

Confidencialidad de los datos. Los autores declaran que han seguido los protocolos de su centro de trabajo sobre la publicación de datos de pacientes.
Derecho a la privacidad y consentimiento informado. Los autores declaran que en este artículo no aparecen datos de pacientes.

\section{Conflicto de intereses}

Los autores declaran no tener ningún conflicto de intereses.

\section{Bibliografía}

1. Berrocal T, Gayá F, de Pablo L. Aspectos embriológicos, clínicos y radiológicos de la malrotación intestinal. Radiologia. 2005;47:237-51.

2. Von Flüe M, Herzog U, Ackermann C, Tondelli P, Harder F. Acute and chronic presentation of intestinal non-rotation in adult. Dis Colon Rectum. 1994;37:192-8.

3. Jiménez Fuentes M, Costa Navarro D, Zamora Amorós C. Dolor abdominal y malrotación intestinal en el adulto. Rev Esp Enferm Dig. 2013;105:105-6.

4. Gallarín Salamanca IM, Espín Jaime MT, Moreno Puertas ÁE, Salas Martínez J. Malrotación intestinal inusual en paciente adulto. Cir Esp. 2016;94:21-3.

5. Etchevers J, Palermo M, Salvatore MG, Tarsitano F, Villafañe V. Malrotación intestinal en adultos: causa infrecuente de abdomen agudo oclusivo. Rev Argent Radiol. 2008;72:435-8. 